\title{
InSb/CdTe HETEROJUNCTION: SELF-CONSISTENT CALCULATIONS OF THE ENERGY STRUCTURE
}

\author{
P. SoвKowICz \\ Institute of Physics, Polish Academy of Sciences \\ Al. Lotników 32/46, 02-668 Warszawa, Poland
}

\section{J. Singleton}

High Field Magnet Lab. and Res. Inst. for Materials, University of Nijmegen NL6525 ED Nijmegen, The Netherlands

\author{
AND S.K. Greene
}

Cavendish Laboratory, University of Cambridge

Madingley Road, GB-Cambridge CB3 OHE, United Kingdom

Results of self-consistent calculations of the subband energy structure of two-dimensional electron gas in the InSb/CdTe heterojunction are reported. Subband occupations and spin splitting in the absence of magnetic field are presented and compared with results of magneto-transport measurements on high mobility, low carrier density heterojunctions.

PACS numbers: 73.20.Dx

\section{Introduction}

Two-dimensional (2D) systems based on narrow-gap semiconductors (NGS) exhibit many properties absent or significantly weaker in wide-gap systems, e.g. strong nonparabolicity, interband tunneling and large spin splitting, both in magnetic field and in its absence. The origin of these effects lies in a strong interaction between the conduction and valence bands in narrow gap materials.

The 2D narrow gap systems studied so far included Metal-Insulator-Semiconductor (MIS) structures of $\mathrm{InSb}, \mathrm{HgCdTe}, \mathrm{HgMnTe}$; inversion layers at grain 
boundaries in InSb, HgCdTe, HgMnTe and HgCdMnTe. The InSb/CdTe heterojunction offers considerable advantages over the above mentioned systems, especially from the theoretical point of view. The constituent materials have the same crystalline structure and almost the same lattice constants, which should lead to a perfect, strain-free interface. The band structures are also similar, having the same ordering of the bands. The $s p$ momentum matrix elements $P$ are very close in both materials, which suggests that the Bloch basis functions are almost identical. This stands in contrast with the situation in MIS structures where the physical interface structure may be far from perfect, the band structure of the insulator is usually unknown (and, presumably, quite different from the semiconductor band structure). Therefore, the system offers a perfect testing ground for the effective mass approximation used in calculations of the energy structures of 2D systems, especially in the treatment of the boundary conditions at the interface between materials. The interface barrier for the conduction band electrons $(\approx 550 \mathrm{meV})$ is almost twice as high as $E_{\mathrm{G}}(\mathrm{InSb})$, which ensures good localization of the electrons in the 2D inversion layer. Thus the system is in many aspects a "better" version of the previously investigated NGS based MIS structures.

In addition the bulk band structure parameters are well known, whereas in the mixed compounds like $\mathrm{HgCdTe}$ or $\mathrm{HgMnTe}$ one may expect variations of the composition and thus of the band parameters used in calculations.

\section{Experimental details}

The magneto-transport measurements were performed on MBE grown InSb/CdTe heterojunctions with high mobilities $\left(16000-22000 \mathrm{~cm}^{2} \mathrm{~V}^{-1} \mathrm{~s}^{-1}\right.$ and low 2D carrier densities $N_{\mathrm{S}}\left(1.8-5.0 \times 10^{11} \mathrm{~cm}^{-2}\right)$. Depending on the $N_{\mathrm{S}}$ one or two subbands are occupied. Their occupations are deduced from careful study of Shubnikov-de Haas and Hall measurements. The data shows that the first excited subband starts to be populated at $N_{\mathrm{S}} \approx 1.8 \times 10^{11} \mathrm{~cm}^{-2}$ [1].

The spin splitting in the absence of magnetic field has been deduced from studies of low-field magnetoresistance [2]. The zero-field spin splitting for the lowest subband, $\Delta_{0}$, has been calculated from fits to W-shaped magnetoconductance. Due to uncertainty of the scattering mechanisms characteristics, the error in the experimentally deduced values of the splitting is of the order of $10 \%$.

\section{Theoretical model and results}

As in the case of the previous investigations of the energy structure of 2D MIS structures of InSb or $\mathrm{HgCdTe}$ [3-7] the self-consistent calculations are based on the "three-band" model of a narrow gap semiconductor. This model treats the interactions between the $\Gamma_{6}, \Gamma_{8}$ and $\Gamma_{7}$ states of the NGS exactly, but neglects all the influence of the other states. As a result, the Hamiltonian matrix $(8 \times 8)$ is linear in $k$. The model proved quite useful in the analysis of the different 2D structures based on the narrow gap semiconductors, leading to relatively accurate predictions of the subband occupations, cyclotron energies and electron $g$-factors. 
However, due to the uncertainties about the structure of the insulator and the interface region in MIS structures it is not possible to give unique theoretical results for some effects, for example for the zero-field spin splitting $[4,5]$.

The three-band model leads eventually to a set of two coupled differential equations for the envelope functions of the two spin states corresponding to the $\Gamma_{6}$ conduction band [5]. The potential $V(z)$ is connected to the electron wave functions via the Poisson equation. For narrow-gap semiconductors, where many body corrections are small, it is usually given by the Hartree approximation. The eigenequations and the Poisson equation for the confining potential have to be solved self-consistently. The fact that the wave functions depend on the wavevector $k_{\|}$means that, in contrast to the parabolic (wide-gap) semiconductors, wavefunctions for the whole range of $k_{\|}$should be included in calculation of $V(z)$.

Due to the similarity of the band and lattice structures of InSb and CdTe matching of the envelope functions at the interface is quite natural. From the conditions of the continuity of the total wa ve function and current conservation one obtains a unique set of conditions connecting the envelopes and their derivatives on both sides of the boundary. These conditions are different for the two spin states and thus contribute to the zero-field spin splitting.

\subsection{Subband occupations}

The subband occupations calculated for the nominal value of bulk InSb acceptor concentration $N_{\mathrm{A}}=1 \times 10^{16} \mathrm{~cm}^{-3}$ are in general in passable agreement with the experiment, especially for higher values of $N_{\mathrm{S}}$. The first subband, however, starts to be populated only at $N_{\mathrm{S}} \approx 3.2 \times 10^{11} \mathrm{~cm}^{-2}$. By lowering the $N_{\mathrm{A}}$ value one can lower the threshold $N_{\mathrm{S}}$ to $2 \times 10^{11} \mathrm{~cm}^{-2}$ for $N_{\mathrm{A}}=3 \times 10^{15} \mathrm{~cm}^{-3}$ (Fig. 1). Note that some change of $N_{\mathrm{A}}$ from the nominal value may have occured due to interdiffusion etc., and may be different from sample to sample.

\subsection{Spin splitting in the absence of the magnetic field}

There are three main sources of zero-field spin splitting in NGS: $k^{3}$ term arising from the bulk anisotropy, linear- $k_{\|}$term due to asymmetry of the electric field in the 2D system and the splitting due to the presence of the boundary between the different materials (also linear in $k_{\|}$). The three-band model takes the two latter contributions into account directly (not by a perturbation technique). On the other hand, the $k^{3}$ term, which clearly lies outside the scope of the model, is not included here at all, not even as a perturbation. The reason behind this decision follows Rössler's argument that the $k^{3}$ term is of lesser importance in NGS than in e.g. GaAs [8]. A crude estimation gives its value at about $1 \mathrm{meV}$ in the studied samples. The calculated results are in reasonable agreement with the experiment (Fig. 2). Here, the agreement seems better for higher values of $N_{\mathrm{A}}$. It is expected that the inclusion of the $k^{3}$ term would enhance the calculated splittings, but one should be cautious in applying simple methods such as subtraction of the $k^{3}$ term from the data: the three contributions mix the states in different ways and the resulting total splitting is not a simple sum. 

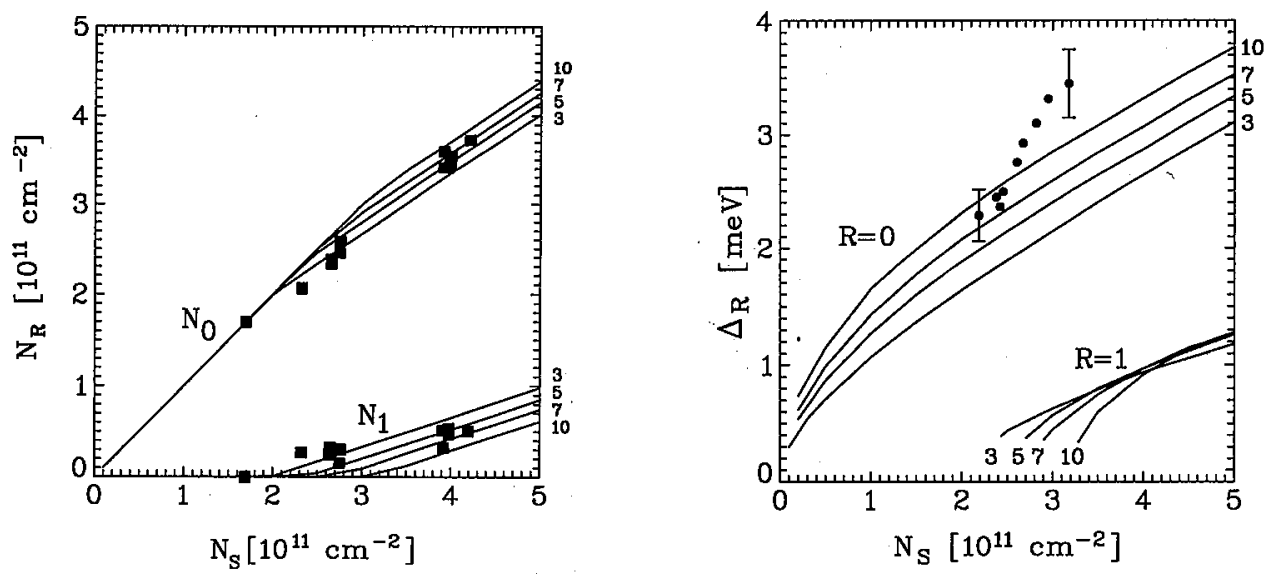

Fig. 1. Comparison of experimental and theoretical subband occupations as functions of total $2 \mathrm{D}$ electron density $N_{\mathrm{S}}$. Lines labelled 3, 5, 7, 10 correspond to bulk InSb acceptor densities of $3,5,7,10 \times 10^{15} \mathrm{~cm}^{-3}$.

Fig. 2. Comparison of experimental and theoretical values of zero-field spin splitting for the two lowest subbands. Lines are labelled as in Fig. 1.

\section{References}

[1] S.K. Greene, J. Singleton, T.D. Golding, M. Pepper, J.A.A.J. Perenboom, J.H. Dinan, to be published.

[2] J. Singleton, S.K. Greene, T.D. Golding, M. Pepper, C. Skierbiszewski, P. Wiśniewski, P.J. van der Wel, P.H.E. van Thor, J. Dinan, Superlattices Microstruct. 9, 51 (1991).

[3] P. Sobkowicz, Proc. 19th Int. Conf. Phys. Semicond., Ed. W. Zawadzki, Institute of Physics, Polish Academy of Sciences, Warszawa 1988, p. 475.

[4] P. Sobkowicz, J. Cryst. Growth 101, 337 (1990).

[5] P. Sobkowicz, Semicond. Sci. Technol. 5, 183 (1990).

[6] I. Nachev, Semicond. Sci. Technol. 3, 29 (1988).

[7] I. Nachev, Phys. Scr. 37, 825 (1988).

[8] G. Lommer, F. Malcher, U. Rössler, Phys. Rev. Lett. 60, 728 (1988). 